\title{
Detect Wormhole attack and Application of Wireless Sensor Network
}

\author{
C. Rajeev, K. Kumar Swamy
}

\begin{abstract}
Wireless Sensor Networks a segment of inescapable processing, are by and by getting utilized on a huge scale to observe genuine period ecological status. This assault bigly affects remote systems, especially against the steering conventions. Steering components can be puzzled and upset while steering control messages are burrowed to misguided course. The passage work between the two scheming assailants is summoned as wormhole interface. In any case, these sensors work under extraordinary vitality imperatives and planned by remembering an application WSN were clench hand utilized in military missions. The structure of a WSN depends on a very basic level on the application, and it must consider factors like the earth, the application's structure, goals, cost, equipment, and framework limitations. The most downside is that the vitality imperative since it seems unfeasible to change or revive the battery. Numerous applications need start to finish solid data transport with clog control to accomplish an alleged exhibition, especially all through overwhelming traffic. The objective of overview is to display a far reaching audit of the ongoing writing in WSN. This paper surveys the principal improvement and new investigation Applications, Evaluation and portrayals examination bearings during this territory.
\end{abstract}

File terms: Wireless Sensor Network, FPGA, Protocols, Sensor arrange administrations, Sensor organize sending, Survey sensor hub, Active Node, CCU.

\section{INTRODUCTION}

Remote sensor systems (WSN's) comprise of countless small sensor hubs that ceaselessly screens natural conditions. Sensor hubs perform distinctive critical errands as sign preparing, figuring, and system self-arrangement to expand organize inclusion and reinforce its flexibility. The sensors all together give overall circumstance of the conditions that offer a bigger number of information than those given by freely working sensors. They are additionally answerable for identifying condition and transmission information. Regularly the transmission task is basic as there is immense measure of data and sensors gadgets are restricted. As sensor gadgets are obliged the system is introduced to assortment of assaults. Customary security components are not pertinent for WSNs as they are normally significant and hubs are limited. Likewise these components don't get rid of peril of some other assaults.

WSNs are important in various fundamental regions like condition, industry, military, social insurance, security and numerous others. For a case, in a military activity, a remote sensor arrange screens various exercises. On the off chance that an occasion is identified, these sensor hubs sense it and

Revised Manuscript Received on September 22, 2019

K. Kumar Swamy, Assistant Professor, Department of Computer Science and Engineering, Malla Reddy Engineering College for Women, Maisammaguda, Hyderabad-500100

Emails:kankala.kumar24@gmail.com report the data to the base station (called sink) by speaking with different hubs. To accumulate data from WSNs, base stations are normally used. They as a rule have more assets (for instance calculation power and vitality) than common sensor center points which have pretty much such requirements. Collection focuses accumulate information from neighboring sensor hubs incorporate the information and forward them to base stations, where the information are additionally handled or sent to a preparing focus. Along these lines, vitality can be protected in WSNs and system life time is in this manner drawn out.

\section{CONCEPTS AND TERMINOLOGY}

In wormhole assault, an assailant structures at least two malevolent hubs in the system at different zones. These hubs are related with the help of low inertness connect. Due to thusly at any rate two vindictive hubs make a more significant level virtual burrow in the system. This virtual passage is used for sending the parcels between the end purposes of passage. At whatever point transmission begins between sources to goal, foe attempt to record transmitted bundles at one area in the system and passages all caught parcels to another area. The wormhole assault is possible paying little respect to whether the assailant has not exchanged off with any hosts and paying little heed to whether all correspondence gives validness and secrecy. The wormhole assault can frame a certifiable danger in remote systems, especially against numerous impromptu system steering conventions and territory based remote security frameworks. We can comprehend wormhole assault with the help of model Acknowledge that, we have two systems An and B. These two systems, An and B, have one malevolent hub X and Y. These two hubs $\mathrm{X}$ and $\mathrm{Y}$ are vindictive hubs, called as wormhole hubs that are connected through a wormhole association. As a result of this association, hubs $\mathrm{X}$ and $\mathrm{Y}$ consider as neighbors for sending directing messages. The aggressor can upset trades between the system An and B. During transmission, the courses in the system can be altered when the assault occurs during convention exposure arrange. We can likewise observe that parcels from convention disclosure stage will get from hub A to hub B speediest if experiencing the wormhole connect since it has minimal number of jumps. This causes the interference of the directing convention and carries extreme harm to the system. Wormhole assault is a one sort of assault for Wireless systems. Where in any event two aggressors are related by quick off-channel interface called wormhole connect In wormhole assault, a few assailants structures 'burrows' to trade the data parcels and replays them into the system.

Published By:
Blue Eyes Intelligence Engineering
\& Sciences Publication




\section{Detect Wormhole attack and Application of Wireless Sensor Network}

The figure exhibits the working of wormhole assault. Bundles got by hub $\mathrm{X}$ is replayed through hub $\mathrm{Y}$ and the a different way. Usually it take barely any bounces for a parcel to cross from a territory close to $\mathrm{X}$ to a region close to $\mathrm{Y}$. Bundles transmitted close to $\mathrm{X}$ experiencing the wormhole will get in contact at $\mathrm{Y}$ before parcels experiencing different bounces in the system. The aggressor can make An and B believe that they are neighbors by sending steering messages, and after that explicitly drop data messages to disturb correspondence among An and B.

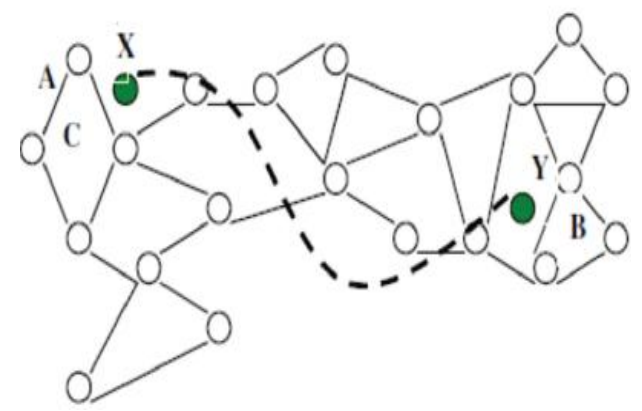

Figure 1: Wormhole Attack

\section{WRITING SURVEY}

$\mathrm{Hu}$, Perrig and Johnson portrayed the wormhole assaults in adhoc systems [1]. A while later, they proposed a system, called bundle rope, which shields parcels from voyaging more than transmission extend. This component depicts two kinds of rope: Geographical and Temporal. In Geographical Leashes, every hub knows its exact area and all hubs have approximately synchronized tickers to decide the neighbor connection. Prior to sending a bundle, hub joins its present position and transmission time to it. On getting parcel, tolerating hub process the separation regarding the sender and the time required by the bundle to cross the way. The gatherer can use this separation data to finish up whether they got parcel experienced a wormhole or not. In Temporal Leashes, each hub keeps up a firmly synchronized clock however doesn't rely upon GPS data [2]. The two components use lightweight hash chains to confirm the hubs [3]. The Message Authentication Code can be resolved continuously. One preferred position of parcel rope is the low calculation overhead.

Melody et al. proposed a wormhole disclosure component reliant on measurable investigation of multipath steering. Tune see that a passage made by a wormhole is amazingly alluring as far as directing, and will be picked and asked with unnaturally high recurrence as it just uses steering data authoritatively open to a hub. These components empowers for simple mix of this strategy into interruption recognition frameworks just to steering conventions that are both on-request and multipath [1].

$\mathrm{Hu}$ and Evans suggested the methodology of directional recieving wires [4]. It depends on the way that in adhoc systems with no wormhole connect, on the off chance that one hub sends parcels in a provided guidance, at that point its neighbor will get that bundle from the other way. Just when the headings are coordinating two by two, the neighboring connection is affirmed. It is basic that each hub requires an excellent equipment for example directional recieving wire. Message voyaging time information is evaluated as far as full circle time (RTT). One way to deal with forestall wormhole assault, as used by Tran et al. [2], Jane Zhen and Sampalli [1], is to quantify RTT of a message and its affirmation. The RTT is the time that connects from the Route Request (RREQ) message sending time of a hub A to Route Reply (RREP) message accepting time from a hub B. Hub A will ascertain the RTT among An and every one of its neighbors. Since the RTT between two fake neighbors is higher than between two certified neighbors, hub A can perceive both the fake and veritable neighbors. In this instrument, every hub registers the RTT among itself and all of its neighbors. No extraordinary equipment is required in this system [1].

Another critical strategy for distinguishing and secluding malevolent hubs that make a wormhole in the system is Trust Based Method by Jain and Jain [1] .In this procedure, trust levels are resolved in neighboring hubs reliant on their validity in execution of the steering convention. This decided trust is then used to affect the steering choices, which thus direct a hub to maintain a strategic distance from correspondence through the wormholes. Accepting that wormholes drop every single bundle it gets, it ought to have least trust level and in this manner can be viably cleared out. By using Trust Based Model Packet Dropping is diminished by $15 \%$ without using any cryptography system and throughput is extended up to $7-8 \%$.

\section{SENSOR NETWORK APPLICATIONS}

Application Development - From Battlefield to Human Body Placing sensors in various territories to assemble physical data for later examination licenses present registering to get practical. Different military-explicit applications have changed into following apparatuses to recognize common data. Progressed pertinent advances are developing, and subsequently, sensors may be embedded in the human body. Sound Surveillance System [8] is that the underlying evident sensor systems application [40]. It had been utilized all through the Cold War inside the mid 1950s to sight and track Soviet submarines with the assistance of acoustic sensors or hydrophones. The Distributed Sensor Networks program was then started by the Defense Advanced Research Projects Agency around 1980. The likelihood to expand the Arpanet to Sensor Networks was viewed as together with some investigation on supporting components like working framework and information based sign handling methods [9]. Application Categorization

There are numerous endeavors to sort sensor arrange applications [10,11]. Every one of them customarily focus on the region of the application getting utilized like wellbeing or natural checking. During this report, two orders are given as pursues:

- Traditional Categorization - Eight sorts of utilization are recorded. This sort mirrors the use of sensor systems for each particular reason. 


\section{Applications of WSNs}

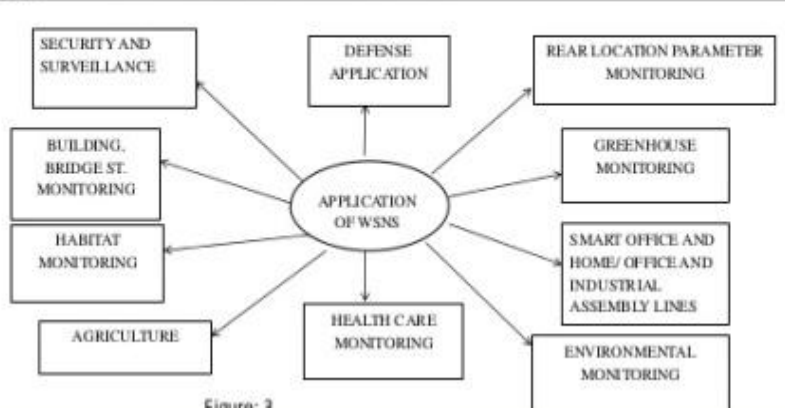

Figure 2: Applications of WSN

- Objective-Oriented Categorization - five classes of use; Military, Public Security/Warning, Education, Business Competitiveness Improvement, and Quality-of-Life Improvement additionally are given. Some conventional applications will be set into more than one class.

\section{SENSOR NETWORK COMPONENTS}

The principle parts of sensors comprise of a detecting unit, a handling unit, a handset, and a power unit as appeared in outline. Each component can be spoken to inside the following segments.

Figure 3: Components of WSN

5.1. Detecting Unit

The fundamental usefulness of the detecting unit is to detect or physical data from the TARGET condition. The simple voltage or sign is produced by sensor comparing to the watched wonder. The nonstop waveform is digitized by a simple to computerized convertor at that point conveyed to the preparing unit for further research [20]. The detecting unit could be present innovation bottlenecks as aftereffects of the detecting advancements are more slow than those of the semi-conductors [21].

\subsection{Preparing Unit}

The preparing unit assumes a critical job in overseeing coordinated effort with various sensors to accomplish the predefined undertakings. There are by and by numerous groups of this unit together with microcontrollers, chip, and field-programmable entryway exhibits [19]. FPGAs devour extra vitality and weren't perfect with conventional programming philosophies. Nonetheless, they'll be reprogrammable and reconfigurable to take out arrangement costs [22].

5.3. Handset

There are sending correspondence conspires in sensors including optical correspondence (laser), infrared, and radiofrequency (RF). Laser expends less vitality than radio and gives high security, anyway needs viewable pathway and is delicate to climatic conditions. Infrared, similar to laser, needs no radio wire anyway is constrained in its telecom ability. RF is that the most simple to utilize anyway requires recieving wire.

Different vitality utilization decrease strategies are created like as regulation, separating, and demodulation. Adequacy and FM are standard instruments. Plentifulness balance is simple anyway vulnerable to commotion [20]. The RF Monolithic TR1000 and 1000 are business radios and wide used in various applications [20,22]. Chipcon 1000 is all the more essentially customized for activity at frequencies somewhere in the range of 300 and $1000 \mathrm{MHz}$ [22].

5.4. Power Unit

Power utilization could be a significant shortcoming of sensor systems. Any vitality conservation plans can expand sensor's lifetime. Batteries utilized in sensors will be arranged into two gatherings; battery-powered and non-battery-powered. As a rule in unforgiving conditions, it's impractical to revive or change A battery. Current sensors are created to have the ability to energize their vitality from sun oriented based or vibration vitality [20,21]. Soluble batteries have a wide voltage extend and gigantic physical size while lithium furnishes a consistent voltage supply anyway with horrendously low ostensible release flows. Nickel Metal Hydride will be revived anyway with a significant lessening in vitality thickness [20].

\section{ASSESSMENT}

Remote detecting innovation containing free, fueled hubs is pushing detecting to the extraordinary. Sensor modules, bits, and ICs all have enormously affected the business as parts of wide going remote sensor systems. During this segment we will in general think about the arranging of WSN sensor hubs anticipated by a few of examination groups. The correlation is generally founded on following specialized highlights.

6.1 Target Technology

In this area assess the bits upheld the consequent specialized highlights for example Reception apparatus, parts, stockpiling, and power. The powerful scope of bits is totally delineated by the radio wire structure. the basic range is in the middle of $100 \mathrm{ft}$ (30.48 meters) to $250 \mathrm{ft}$ (76.2 meters) at the same time, there are barely any bits like Mica2 and Iris that may give $1000 \mathrm{ft}$ accessible range capacity and this may be useful for a wide inclusion territory anyway then again bigger the inclusion space the lesser are the normal life time of the WSN hub $[9,12]$ hence exchange off between the hub life time and inclusion are s ought to be mulled over all through the segment of WSN hubs while arranging a WSN organize. 6.2 Communication Technology

While structuring a WSN the architect should give key consideration to the existence time of the entire system since one among the most goal of WSN is to have least human intercession Other than handling the correspondence part is viewed as the second biggest vitality expending segment of the hub. The RF handset expends the vast majority of the vitality all through the dynamic state. System period will be expanded by having hubs just work their radios for brief timeframes.

\section{PROPOSED WORK}

Wormhole assaults are carefully identified with organize layer conventions. As new directing conventions are proposed for WSNs, it fundamental to perceive possible insufficiencies of these new steering conventions, measure the exhibition of new directing convention with wormhole assault and to investigate the ampleness of the present wormhole acknowledgment systems on these conventions. 


\section{Detect Wormhole attack and Application of Wireless Sensor Network}

Consequently, there is an expansion for further research similarly as assessing execution of existing wormhole distinguishing proof techniques on new steering conventions. Future work here centers around additional security upgrades for steering conventions in remote sensor systems.

The wormhole recognition in a dynamic WSN is an open research region. In a dynamic WSN, any two real sensor hubs that were already numerous bounces a long way from one another may become one jump neighbors, and thus makes dream for the base station that a wormhole assault has been propelled. Subsequently, it is a moving undertaking to perceive such bona fide hubs from malevolent hubs while distinguishing wormhole assaults.

I might want to build up another methodology which identifies wormhole assault as the examination work in the territory of Wireless Sensor Networks.

VI. End

This report expects to give a few audits of remote sensor systems. Some investigation data concerning the system applications, segments, solid transport conventions and clog control approaches are given. Sensor systems are troublesome in being small, incredible anyway with less vitality utilization, still as obliging entirely unexpected application needs. Further, the vehicle convention assumes a noteworthy job in furnishing start to finish correspondence with blockage control. tcp doesn't seem ready to give dependable data transport in sensor systems. A solid transport convention with clog control capacity for sensor systems is should have been nonexclusive, light-weight, and not reliant after existing lower layer conventions.

WSN could be a promising future innovation and directly utilized in scope of use that requirements least human mediation. During this paper we have studied the WSN innovation. We have likewise exhibited the WSN mode investigation bolstered its key specialized particulars. In spite of the fact that specialists have just planned assortment of system designs like heterogeneous and single-jump that utilizations WSN innovation anyway in such arrangements the greater part of the preparing is done at the server end. It would be keen if in-arrange handling capacity will be consolidated at hub's end. By thusly the hub preprocesses the information and sends remotely the conservative type of the separated information to the sink.

\section{REFERENCES:}

1. Preeti Nagrath, Bhawna Gupta, "Wormhole Attacks in Wireless Adhoc Networks and their Counter Measurements: A survey", pp 245-250, IEEE 2011.

2. Majid Meghdadi, Suat Ozdemir and Inan Guler, "A Survey of Wormhole based Attacks and their Countermeasures in Wireless Sensor Networks", IETE TECHNICAL REVIEW, VOL 28, ISSUE 2, Mar-Apr 2011.

3. Dhara Buch, Devesh Jinwala, "Detection of Wormhole Attacks in Wireless Sensor Networks", IEEE Conference on Advances in Recent Technologies in Communication and Computing, pp 7-14, 2011.

4. Zhibin Zhao, Bo Wei, Xiaomei Dong, Lan Yao, Fuxiang Gao, "Detecting Wormhole Attacks in Wireless Sensor Networks with Statistical Analysis", IEEE International Conference on Information Engineering, pp 251-254, 2010.

5. Zaw Tun et al., "Wormhole Attack Detection in Wireless Sensor Network" World Academy of Science, Engineering and Technology 46 2008.

6. Dr.S.P.Anandaraj " Research Opportunities and Challenges of a Security Concerns associated with Big Data in Cloud Computing”, IEEE International conference on ISMAC IoT in Social, Mobile, Analytics and Cloud published in IEEE Explore,
DOI: 10.1109/I-SMAC.2017.8058278,

ISBN: 978-1-5090-3243-3,Print ISBN: 978-1-5090-3242-6, INSPEC Accession Number: 17224835 on 10th to 11th Feb, 2017

7. Yurong $\mathrm{Xu}$ et al., "Detecting Wormhole Attacks In Wireless Sensor Networks" Critical Infrastructure Protection, Chapter 14.

8. .J. Pike, "Sound Surveillance System (SOSUS),"[Online] Available at http://www.globalsecurity.org/intell/systems/sosus.htm, November 2002.

9. Dr.S.P.Anandaraj, S.Poornima, Sougandhika Reddy,M Sindhuja,P.Vedhakshatha, "Semantic Analysis On Communication And Security Issues Of Extensible Authentication Protocol (EAP) On Wireless Networks" International Journal of Advanced Computing and Electronics Technology(IJACET), ISSN(P):2394-3408,(O):2394-3416,Vol 2, No 1, January 2015

10. Chee-Yee Chong, and S.P. Kumar, "Sensor Networks: Evolution, Opportunities, and Challenges," Proceedings of the IEEE, Vol. 91, No.8,August 2003.

11. N. Xu, "A Survey of Sensor Network Applications," Survey Paper for CS694a, Computer Science Department, University of Southern California.

12. C. W. Fu, Bang. Lim, Hock Beng. , "An Enhanced Sensor Scheduling Protocol for Wireless Sensor Networks," in Distributed Comp Sys Workshops, 2008. ICDCS '08. 28th Int Conf on, Beijing, China 17-20 June 2008, pp. 303-308.

13. MIT WSN Research Group: http://mtlweb.mit.edu/researchgroups/icsystems/gallery.html, Aug. 20, 2008 .

14. U. W. Bilstrup, P.A, "An architecture comparisonbetween a wireless sensor network and an active RFID system," in Local Computer Networks, 2004. 29thAnnual IEEE International Conference on, Halmstad University, CERES, Nov. 2004, pp. 583- 584.

15. Y.-I. Y. Kim, Bong-Jae. Song, Jae-Ju. , "Implementing a Prototype System for Power. Facility Management using RFID/WSN," pp. 70-75, in IADIS International Conference WWW/Internet SPAIN, 2006

16. N. Cho, S.J. Song, S. Kim, S. Kim and H. J. Yoo, "A 5.1 microwatt UHF RFID Tag chip integrated with sensors for wireless environment monitoring" Proc of the ESSCIRC Grenoble, France (2005).

17. METRANS Transportation Center, "SURE-FT: Sensor for Unexpected Roadway Events: Field Trials," [Online] Available at http://www.metrans.org/ Research /current_proj/05-14.htm.

18. N. Tatbul, M. Buller, R. Hoyt, S. Mullen, and S. Zdonik, "Confidence-Based Data Management for Personal Area Sensor Networks," Proceedings of the First Workshop on Data Management for Sensor Networks (DMSN 2004), Toronto, Canada, August 2004.

19. Dr.S.P.Anandaraj, D.Naveen Kumar, S.Poornima, "Invariant Character View Recognition in Artificial Neural Networks based on pattern classification" Global Journal of Computer Science and Information Technology (GJCSIT), Vol 1, No.1,pp. 33-37, Oct 2014.

20. Jason Lester Hill, "System Architecture for Wireless Sensor Network," PhD Dissertation, University of California, Berkeley, 2003.

21. J. Feng, F. Koushanfar, and M. Potkonjak, "System-Architecture for Sensor Networks Issues, Alternatives, and Directions,” ICCD'02, 2002.

22. M.A.M. Vieira, C.N. Coelho. Jr., D.C. da Silva Jr., and J.M. da Mata, "Survey on Wireless Sensor Network Devices," IEEE, 2003.

23. N. Nithiyanandam, K. Venkatesh, M. Rajesh, Transfer The Levels Of The Monitored Carbon, Nitrogen Gases From The Industries, International Journal of Recent Technology and Engineering, Volume-7 Issue-6S3 April, 2019.

24. Sivanesh Kumar, A., Brittoraj, S., Rajesh, M., Implementation of RFID with internet of things, Journal of Recent Technology and Engineering, Volume-7 Issue-6S3 April, 2019.

25. Rajesh, M., Sairam, R., Big data and health care system using mlearningJournal of Recent Technology and Engineering, Volume-7 Issue-6S3 April, 2019.

26. Rajesh, M., and J. M. Gnanasekar. "Path Observation Based Physical Routing Protocol for Wireless Ad Hoc Networks." Wireless Personal Communications 97.1 (2017): 1267-1289. 